\title{
A Thermal Desorption Spectroscopy Study of Hydrogen Trapping in Polycrystalline $\alpha$-Uranium
}

\author{
R.S. Lillard*, R.T. Forsyth ${ }^{a}$ \\ Department of Chemical and Biomolecular Engineering \\ University of Akron \\ Akron OH 44325
}

\begin{abstract}
The kinetics of hydrogen desorption from polycrystalline $\alpha$-uranium $(\alpha-U)$ was examined using thermal desorption spectroscopy (TDS). The goal was to identify the major trap sites for hydrogen and their associated trap energies. In $\alpha-U$ six TDS adsorption peaks were observed at temperatures of 521K, 556K, 607K, 681K, 793K and $905 \mathrm{~K}$. In addition, the desorption was determined to be second order based on peak shape. The position of the first three peaks was consistent with desorption from $\mathrm{UH}_{3}$. To identify the trap site corresponding to the high temperature peaks the data were compared to a plastically deformed sample and a high purity single crystal sample. The plastically deformed sample allowed the identification of trapping at dislocations while the single crystal sample allow for the identification of high angle boundaries and impurities. With respect to the desorption energy associated with each peak, values between 12.9 and $26.5 \mathrm{~kJ} /$ mole were measured.
\end{abstract}

key words: alpha uranium, hydride, trap site, thermal desorption spectroscopy, TDS, activation energy, single crystal

*corresponding author: lillard@uakron.edu

a MST-6, Materials Science and Technology Division, Los Alamos National Laboratory, Los Alamos, NM 87545 


\section{Introduction}

Naturally occurring uranium is composed of three isotopes: uranium-238, (0.7\%) uranium-235 (99.3\%), and uranium-234 (approx 0.005\%). For this study depleted $\alpha$-uranium $238\left({ }^{238} \mathrm{U}\right)$ was examined (less than $0.3 \%{ }^{235} \mathrm{U}$ ). Depleted $\alpha$ uranium 238 is the common engineering isotope in both defense and civilian applications and is attractive because of its density in the manufacturing of armor plating, projectiles, radiation shielding and the counter weights / ballast used in aircraft. In these applications it is often exposed to hydrogen environments either in the form of gaseous hydrogen or hydrogen that results from water reduction (e.g. the cathodic reaction during uranium corrosion). This hydrogen uptake results in shorten service lifetimes due to uranium hydride $\left(\mathrm{UH}_{3}\right)$ formation resulting in metal loss and volumetric expansion as $\mathrm{UH}_{3}$ has a lower density than the metal. Hydrogen uptake is also know to cause embrittlement in uranium.

Both the thermodynamics and kinetics of the $\mathrm{U}$ to $\mathrm{UH}_{3}$ phase transformation process in powders have been experimentally characterized [1-5] and the focus of first principle models.[6,7] Removal of hydrogen (and $\mathrm{UH}_{3}$ ) from metal, however, has been less studied but has practical importance for parts that will be recycled.[8] For example, the kinetics of hydrogen decomposition controls the soak-time at a given temperature necessary to remove a desired fraction of $\mathrm{H}$. In a previous publication the kinetics of powder $\mathrm{UH}_{3}$ decomposition were described using thermal desorption spectroscopy (TDS).[9] In TDS, the sample to be investigated is placed in a HV (high vacuum) chamber equipped with a furnace and a residual gas analyzer (RGA). The sample is heated (either isothermally or at a given ramp rate) 
by the furnace and the corresponding spectrum of gases that evolve with time are analyzed individually using the RGA. The resulting $[\mathrm{H}]$ vs temperature plots typically contain multiple peaks, each associated with the energy required to release hydrogen from a given state.

To understand the assumptions associated with TDS measurements and data analysis, consider the energy diagram in Figure 1 associated with the trapping of hydrogen in a metal proposed by Griffiths and adopted by Flanagan.[10,11] In the absence of defects, once absorbed hydrogen is transported in the metal by interstitial diffusion (also referred to as lattice diffusion). The activation energy necessary to transport a hydrogen atom from one interstitial site to another (diffusion) is denoted by $E_{D}$ in this figure. As the hydrogen diffuses through the metal it may encounter any of a number of trap sites such as second phase particles, voids, twin boundaries, grain boundaries, etc. Each of these trap sites are associated with a binding energy, $E_{A}$. For any site with a binding energy greater than $E_{D}$, the energy required for desorption $E_{d e s}$, is equal $E_{D}+E_{A}$ By heating the sample it is possible to increase the system energy above $E_{\text {des }}$ to remove hydrogen from a trap site. In the TDS experiment where the temperature is ramped from low to high the release of hydrogen from the metal is associated with a peak in the RGA current for mass 2 where the desorption flux (rate) is proportional to the concentration gradient and diffusivity. The area under the peak is proportional to the concentration of hydrogen trapped at the site. This description of hydrogen trapping is of a first order reaction, though other reaction orders may be possible. 
For example, in a second order reaction there may be intermediate trapping states that result when hydrogen interacts within the lattice. Reaction order is frequently determined from the shape of the RGA peak with first order reactions having a sigmoidal (non-uniform) peak shape and second order reactions having a Gaussian peak shape (uniform).[12] The location of temperature maximum $\left(\mathrm{T}_{\mathrm{m}}\right)$ is related to $E_{\text {des. }}$ The change in peak temperature with heating rate enables the calculation of $E_{\text {des }}$ using the relationship[13,14]:

$$
\frac{E_{d e s} \phi}{R T_{m}^{2}}=k_{0} n \theta^{n-1} \exp \left\{-\frac{E_{d e s}}{R T_{m}}\right\}
$$

In this expression $\phi$ is the temperature ramp rate in $\mathrm{K} / \mathrm{min}, T_{m}$ is the temperature of the maximum hydrogen desorption rate in $\mathrm{K}, R$ is the ideal gas constant and is equal to $8.314 \mathrm{~J} / \mathrm{K} \cdot \mathrm{mol}, E_{\text {des }}$ is in $\mathrm{J} / \mathrm{mol}, \mathrm{n}$ is the reaction order, $\theta$ is the fraction hydrogen coverage of the trap and $k_{0}$ is a pre-exponential factor with units of min $^{-1}$.

In a previous publication, we described the TDS spectra for $\mathrm{UH}_{3}$ powders. It was shown that the TDS spectra for $\mathrm{UH}_{3}$ contained three desorption peaks and Eq. 1 was used to measure an activation energy for decomposition of $43 \mathrm{~kJ} / \mathrm{mol}-\mathrm{H}$ for the decomposition of the powder.[9] The mechanism responsible for desorption in $\mathrm{UH}_{3}$ powders was discussed within the context of an $a b$ initio model. In addition, it was also shown that hydride corrosion pits in $\alpha$-uranium metal $(\alpha-U)$ contained six desorption peaks. However, no formal analysis of that data was conducted and the mechanism responsible for the uptake of hydrogen in $\alpha-U$ was not investigated. The goal of this investigation is to identify the major trap sites (microstructural defects) 
responsible for the desorption peaks in $\alpha$-U. Identification of the microstructural defects associated with these peaks affords engineers the opportunity to design a metal/alloy that is free of the defect site and, thus, reduce the damage associated with hydrogen uptake. An additional goal of this investigation is to measure the trap energies associated with each defect in $\alpha-U$. From an engineering perspective, trap energies can be used to calculate desorption profiles and determine minimum time needed to remove hydrogen from a component at a given temperature so that its service life may be extended.

\section{Experimental}

\subsection{Sample Preparation and Characterization}

To investigate the hydrogen degradation of $\alpha$-U we have examined both polycrystalline and a single crystal material. The polycrystalline $\alpha$-U cast rod stock was 98.5 wt.\% $\mathrm{U}$ with remaining 1.5 wt.\% consisting of $\mathrm{C}$ and $\mathrm{O}$ and a grain size ranging between $0.5 \mathrm{~mm}$ in the center of the rod and $5 \mathrm{~mm}$ at the edges. From the

rod stock, samples were cut in the form of discs measuring approximately $0.9 \mathrm{~cm}$ in diameter by $0.09 \mathrm{~cm}$ thick and weighing $0.89 \mathrm{~g}$. Polycrystalline samples were prepared by polishing to $1 \mu \mathrm{m}$ diamond and then electropolishing in $5 \mathrm{wt} \%$ orthophosphoric acid for approximately $5 \mathrm{~s}$ at $1.7 \mathrm{~V}(100-200 \mathrm{~mA})$. The single crystals of $\alpha-\mathrm{U}$ were grown from a low temperature molten salt bath of $\mathrm{LiCl}-\mathrm{KCl}$ eutectic containing $3 \mathrm{wt} . \% \mathrm{UCl}_{3}$ and had a nominal C concentration of $<50 \mathrm{ppm}$.[15] Because the uranium was deposited at temperatures below the $\alpha$ - to- $\beta$ transformation 
temperature, the single crystals were strain free. They were nominally parallelograms where (100) orientation represented the largest fraction of the surface area and measured approximately $0.58 \times 0.66 \times 0.09 \mathrm{~cm}$ and weighed $0.21 \mathrm{~g}$. Single crystal samples were prepared by electropolishing only. Following the electropolishing step, the polycrystalline and single crystal samples were electrochemically charged with $\mathrm{H}$ in the electropolishing solution (without removal). Hydrogen charging was done electrochemically using a galvanostat and a constant current density of $-3.2 \mu \mathrm{A} / \mathrm{cm}^{2}$ for approximately $1800 \mathrm{~s}$. This procedure was found to eliminate the formation of hydride pits, however, by the end of charging period a thin transparent film (tan) had formed on the sample that was, presumably, "hydrogen rich." This is further discussed below.

To capture the composition and other characteristics such as inclusions in the samples, scanning electron microscopy (SEM) analysis was performed using both backscatter and secondary electrons. Additionally, energy dispersive spectroscopy (EDS) was used to map the elemental composition of the inclusions. The inclusions were specifically mapped for uranium, carbon, oxygen, and nitrogen. Statistical information regarding inclusion size was conducted using a series of $100 \mathrm{x}$ (polycrystalline) and 250x (single crystal) digital images using commercially available image analysis software. For the single crystal sample a total of nine areas were chosen at random for a total area analyzed of $0.0055 \mathrm{~cm}^{2}$. As the polycrystalline sample was larger, a total of 15 areas were chosen at random for a total area analyzed of $0.58 \mathrm{~cm}^{2}$. In the analysis, a software routine was created to 
differentiate the inclusions from the matrix using a grayscale. The inclusions were assigned to a specific bitplane, artifacts were removed and the bitplane representing the inclusions was measured. Two types of measurements were obtained, field measurements and object measurements. Field measurements relate the inclusions to the overall image or field. Field measurements were taken for average area, area percentage, and density. Object measurements, taken on individual inclusions, were taken for area and equivalent circular diameter. Object measurement data for any inclusions that intersected the edge of the image were filtered from the final data.

\subsection{Thermal Desorption Spectroscopy}

A diagram of the TDS system used in these experiments is depicted in previous publications.[9] It was constructed of SS 304L and consisted of two connected vacuum chambers: one for sample loading/heating and a second for gas analysis (base pressure of $1 \times 10^{-8}$ to $3 \times 10^{-8}$ torr, active pumping). Sample temperature was controlled using a tube furnace that surrounded a quartz tube used to hold the sample and a PID controller. The volume of the sample side of the system was found to be $0.91 \mathrm{~L}+/-0.02 \mathrm{~L}$. In the analysis side of the chamber, an RGA was used to quantify the amount of hydrogen generated during heating of the sample. The RGA was calibrated using a set of Leco hydrogen standards and the relationship between the area under the current-time curve for those standards was related to the mass of $\mathrm{H}$ by the relationship:

$$
\mu g(H)=-1.07+2.76 \times 10^{-7} A
$$


where: $\mu g(\mathrm{H})$ is the mass of atomic hydrogen in micrograms and $A$ is the area below the current-time curve in Amp·s.[9]

\subsection{STM Measurements}

In situ electrochemical scanning tunneling microscopy (EC-STM) was performed using a Veeco Nanoscope III and Apezon coated Pt-Ir tips (Agilent) using a specially designed electrochemical cell equipped with a Pt wire counter electrode and an Ag wire reference electrode. EC-STM experiments were conducted under potentiostatic control at potentials cathodic of the $U$ open circuit potential cell currents $<10 \mu \mathrm{A} / \mathrm{cm}^{2}$ and a tunneling set point of $500 \mathrm{pA}$. The solution used in these experiments was 2 wt\% o-phosphoric acid.

\section{Results and Discussions}

\subsection{Hydrogen Desorption from Polycrystalline $\alpha-U$}

Results from a thermally ramped TDS experiment on polycrystalline $\alpha-U$ are shown in Figure 2a. The data were generated for a ramp rate of $5 \mathrm{~K} / \mathrm{min}$ and represent the typical shape of all plots with lower ramp rates being shifted to higher temperatures and higher ramp rates shifted to lower temperatures. As seen in this figure, the measured TDS data appear to be the summation of desorption from multiple trap sites. Specifically, the curves could be modeled as six individual desorption peaks (plus a surface desorption pre-peak at low temperature, $\mathrm{p}$, not discussed here) with each peak being from an independent trap site, as seen in the 
fit of the spectra using Gaussian curves in Figure 2b. The peaks are numbered 1-6 consistent with the data generated in our previous TDS investigation of $\mathrm{UH}_{3}$ powders with increasing peak number being associated with a desorption peak that occurred at higher temperatures. The fact that the curve could be fit using Gaussian peaks indicates that desorption of hydrogen from $\alpha-U$ is second order. Had the desorption reaction been first order, the peaks would have been sigmoidal in shape. A summary of the characteristic temperature $\left(\mathrm{T}_{\mathrm{m}}\right)$ for each peak as a function of ramp rate is presented in Table 1.

While no other literature exists for TDS experiments on $\alpha$-U, Powell investigated isothermal vacuum degassing of high purity uranium (0.01 wt \% C, $0.019 \mathrm{wt} \% 0)$. [8] Although the furnace was held constant at $1173 \mathrm{~K}$, they monitored hydrogen evolution rate as a function of sample temperature. In the resulting spectra, 4-8 hydrogen evolution peaks were observed (no deconvolution was performed by Powell so the exact number is not known) that were attributed to the $\alpha$ to $\beta$ and $\beta$ to $\gamma$ phase transitions in the metal (at $935 \mathrm{~K}$ and $1044 \mathrm{~K}$, respectively). More likely, however, those peaks were associated with the same trap sites present in our samples.

\subsection{Trap Site Identification}

To identify the microstructural defect associated with each peak in the TDS spectra for the polycrystalline sample (Table 1) the data were analyzed with several "standards." In the first set of standards, our prior TDS work on $\mathrm{UH}_{3}$ powders was 
compared to the TDS spectra from polycrystalline samples. As the TDS spectra for $\mathrm{UH}_{3}$ powders represent the decomposition of $\mathrm{UH}_{3}$ only, comparison with the powder data should identify hydride decomposition in the polycrystalline TDS data if present. The TDS data from the polycrystalline $\alpha-U$ sample are compared with those from $\mathrm{UH}_{3}$ powder in Figure 3a. The data were generated for a ramp rate of 5 $\mathrm{K} / \mathrm{min}$ and are typical of other TDS spectra for this sample. In this plot, a parameter proportional to the RGA current at mass 2 is plotted as a function of temperature so that the two curves may be compared directly. As seen in this figure, the two plots are similar at temperatures below approximately $650 \mathrm{~K}$. To better compare these data, the curve fits for each of the plots are presented in Figure 3b. As seen in this plot, below $650 \mathrm{~K}$ there are three peaks in both the polycrystalline and $\mathrm{UH}_{3}$ samples. In addition, there is a one-to-one correlation between Peak 1 and Peak 2 in both spectra with Peak 3 being shifted to slightly higher temperatures in the polycrystalline $\alpha-U$ sample $(22 \mathrm{~K})$. As such, peaks are assigned 1-3 in the polycrystalline $\alpha-\mathrm{U}$ to the formation of $\mathrm{UH}_{3}$. As no $\mathrm{UH}_{3}$ corrosion pits were formed on the sample during electrochemical charging, it is believed that the $\mathrm{UH}_{3}$ is in the form of a surface layer and is responsible for the translucent "tan" color change in the sample during charging described above. Similar $\mathrm{UH}_{3}$ film formation has been observed during gaseous exposure of $\alpha$-U. In that case, the reaction begins with the formation of hydride islands on the surface that grow in size until the entire surface is covered. [16] Ultimately, the layer cracks and spalls from the surface owing the large volume of expansion, however, thickness up to $2.2 \times 10^{-5} \mathrm{~m}$ have been reported.[17] During low current galvanostatic charging as used here, the reaction 
rate appears to be sufficiently slow to allow some diffusion from the surface layer hydride into the bulk. As such, the layer may not be stoichiometric $\mathrm{UH}_{3}$ and, thus, the shift in peak three of the TDS spectra to higher temperatures in the polycrystalline $\alpha-U$ sample. In a previous publication, it has been proposed that Peaks 1 and 2 in $\mathrm{UH}_{3}$ desorption are associated with the formation of vacancies and di-vacancies in the powder.[9] Specifically, it was suggested that the mechanism of $\mathrm{UH}_{3}$ decomposition occurs by the formation of a metallic shell around a shrinking $\mathrm{UH}_{3}$ core. Once sufficient vacancy formation has occurred, phase transformation from a defective hydride to an impure (H-contaminated) metal occurs. Assuming that H-depletion commences at the exterior of the powder particles, this process would result in a $\mathrm{UH}_{3}$ core that was encapsulated by $\mathrm{U}$ metal, e.g. $\mathrm{UH}_{3}$ in a metallic "shell". As vacancy formation in the $\mathrm{UH}_{3}$ core continued, the rate controlling step for dehydriding is the diffusion of hydrogen through the outer metal shell before desorbing, e.g. Peak 3. Recently a shrinking core model has been formalized by Salloum. [18]

In the second set of standards, the TDS data are compared from annealed polycrystalline samples with plastically deformed samples. As plastic deformation is associated with an increase in dislocation density, we hoped to identify the corresponding trap sites. Comparison of the TDS spectra for an annealed polycrystalline $\alpha$-U sample with data from a sample that had been plastically deformed is presented in Figure 4a and the corresponding fit to the data in Figure $4 \mathrm{~b}$. The data were generated for a ramp rate of $5 \mathrm{~K} / \mathrm{min}$ and the sample was 
charged at the same current density and for the same time (e.g. equal charge) as all other samples. Plastic deformation was obtained by cold rolling an annealed sample at $925 \mathrm{~K}$ for 2 hours, [19] to approximately a 5\% reduction in thickness. For strains greater than $3.5 \%$ it is reported that the dislocation density is on the order of $2.3 \times 10^{10} \mathrm{~cm}^{-2}$ and further reduction in area does not result in an appreciable increase from this value.[20] This is approximately a 100 fold increase in the dislocation density at room temperature. An increase in dislocation density should result in an increase in hydrogen trapping at these sites and subsequently an increase in the corresponding TDS peak. As seen in the TDS data from the deformed sample, a large increase in the relative desorption from Peak 1 as compared to peaks 2-7 was observed. Correspondingly this peak must be associated with dislocations, however, this peak is also associated with $\mathrm{UH}_{3}$. Thus, $\mathrm{UH}_{3}$ formation occurs preferentially at dislocations. In the work of Flanagan on the absorption of $\mathrm{H}$ in $\alpha$-Pd and the formation of $\mathrm{PdH}_{0.02}$ (coexistence of $\alpha$ and $\beta$ hydride phases) it was found that cold-work resulted in an increase in the solubility of hydrogen, e.g. the H/Pd ratio in cold worked samples exceeded 0.02.[21] It was further shown that, when a cold worked Pd sample charged with hydrogen to convert it to the $\beta$ phase was outgassed at room temperature the solubility enhancement was still found upon subsequent charging, e.g. the increased $\mathrm{H} / \mathrm{Pd}$ ratio due to cold work was not removed by the hydride phase transformation.

Finally, we examine the data from high purity single crystal $\alpha$-U samples. These single crystal samples allow for the identification of two types of trap sites: 1 - high 
angle boundaries such as grain boundaries and 2 - because they are so pure, no hydrogen trapping will occur at impurities in these samples such as second phase particles. With respect to trapping at impurities, Arkush et al. found that $\mathrm{UH}_{3}$ was formed preferentially at defects such as $\mathrm{UC}_{\mathrm{x}}$ inclusions after exposure to $101 \mathrm{kPa}$ at 323-348 K.[22] As it relates to this study, we have characterized the composition and distribution of inclusions in our samples. The single crystal sample was associated with only a very limited number of widely spaced very small second phase particles that could only be identified during SEM characterization. In comparison, the size and distribution of second phase particles in the polycrystalline material were quite large. A typical SEM image of an inclusion in the polycrystalline material presented in Figure 5a. Energy dispersive spectroscopy determined the dark regions of the inclusions to contain both oxygen and carbon (oxy-carbides, Fig 5b). Furthermore, EDS maps indicated the lighter regions of the inclusions were primarily carbides (Fig. 5c). The distributions of inclusion area and equivalent circular diameter are shown in Figure 6a and 6b. If the maximum in the data are normalized by the total area examined as before we calculate that there are on the order of 520 inclusions per $\mathrm{cm}^{2}$ with an area between $50-150 \mu \mathrm{m}^{2}$.

Typical TDS data from single crystal $\alpha-U$ are presented in Figure 7a and the corresponding fit in $7 \mathrm{~b}$. As before, the data were generated for a ramp rate of 5 $\mathrm{K} / \mathrm{min}$, and the sample was charged at the same current density and for the same time as all other samples. As was the case in the polycrystalline $\alpha$-U sample, 7 peaks were observed in this spectrum. While peak positions in the single crystal are 
shifted to higher temperatures as compared to the polycrystalline sample, the most noticeable difference is the relatively low hydrogen concentrations associated with Peaks 5 and 6 as compared to that in the polycrystalline samples. We propose that these peaks are associated with high angle boundaries at the intersections of grains and inclusions with the matrix.

While it is likely that the most of the hydrogen trapped by inclusions is a bulk phenomenon, it is also possible that inclusions had higher hydrogen reduction rates during electrochemical charging of the sample. In order to determine if the oxycarbide inclusions were electrochemically active we performed in situ electrochemical STM measurements on the polycrystalline sample near an inclusion to examine cathodic reaction rates. Figure 8a shows the in situ EC-STM height map of a $\mathrm{U}^{238}$ sample in $2 \mathrm{wt} \%$ o-phosphoric acid ( $\mathrm{E}=-690 \mathrm{mv}, \mathrm{E}_{\mathrm{T}}=-200 \mathrm{mV}, \mathrm{E}_{\mathrm{B}}=490 \mathrm{mV}$, $\mathrm{i}_{\text {set }}=0.5 \mathrm{nA}$ ). This image was taken under potentiostatic control slightly cathodic of the $U$ open circuit potential (cell current $\approx-6 \mu \mathrm{A} / \mathrm{cm}^{2}$ ). The bias potential was 300 $\mathrm{mV}$ and the tip Faradaic current was less than $0.01 \mathrm{nA}$. The tunneling set point was 0.5nA. The data were collected at an applied electrochemical current slightly cathodic of the open circuit potential, $\mathrm{E}=-500 \mathrm{mV}$ vs. Ag (approx. -0.56 vs. SCE). The total cell current, $\mathrm{i}_{\text {cell }}$ was equal to $-8.6 \mu \mathrm{A} / \mathrm{cm}^{2}$ in the region of hydrogen reduction. In Figure 8b, the feedback loop was adjusted to measure the electrochemical potential between the STM tip and the sample surface for a constant tip - sample separation distance. Thus, the tip potential in this map is not a measure of height, rather, electrochemical current, i.e. the reaction rate of the hydrogen reduction 
reaction. As seen in this figure, the inclusion shows signs of high current density (hydrogen reduction rate) near the inclusion / uranium interface (white edges in Fig. 8b), that appear on both the leading and trailing edges ruling out scan direction effects. Thus, the interface between the oxy-carbide inclusions and the matrix are preferential sites for hydrogen reduction and likely responsible for trapping. While it is not possible from these results to tell which TDS peak is associated with inclusions, investigations of aluminum alloys found that trapping at grain boundaries was associated with higher temperature peaks as compared to inclusions.[23] Based on those results Peak 5 in the polycrystalline sample may be associated with oxycarbide inclusions. By default we assign Peak 6 with grain boundaries. Scott et al. have shown that grain boundaries in polycrystalline $\alpha-U$ were preferential sites for the precipitation of $\mathrm{UH}_{3}\left(\mathrm{UD}_{3}\right.$ in that study).[24] In that study, exposure to $D_{2}$ was optimized to ensure a high nucleation rate of attack sites but to also limit the subsequently linear growth rate such that nucleation sites could be identified. It was proposed that some of this attack pattern might be related to the structure of, and the mode of transport of deuterium species through the surface oxide film at these boundaries. For example, cracking of the oxide at metal grain boundaries due to differential expansion of adjacent metal grains may result in hydride precipitation at those locations.

Finally, it is worth noting that Peak 4 remains relatively unchanged in the single crystal and polycrystalline samples in both the annealed and deformed cases. As such we conclude that the trap site associated with this peak in the single and 
polycrystalline samples must be similar. One can only speculate on the types of defects these three samples share in common, for example, vacancies and low angle boundaries such as twins. Twinning in $\alpha-U$ involves several slip planes, the most common are the (010) [100] and \{130\} slip systems.[25] Hill et al. report on the preferential hydriding in polycrystalline $\alpha$-U along (130).[26] In that investigation electron backscattered diffraction was used to correlate preferred hydride propagation with lattice directions. It was found that most all hydrides were associated with some type of defect in the material. It was concluded that defects such as grain boundaries, inclusions and twins acted as "fast pathways" for hydrogen diffusion. The preferred defect was twin boundaries. It was proposed that the inability of the twin boundary to accommodate strain combined with the preferential diffusion of hydrogen resulted in growth along the boundary.

A summary of the peak position and the trap site it is attributed to from this work can be found in Table 2 .

\subsection{Activation Energy Analysis}

The activation energy of each of the traps is determined using Eq. 1 by taking the natural log of both sides and rearranging to give the expression:

$$
\ln \left(\frac{T_{m}^{2}}{\phi}\right)=\frac{E_{d e s}}{R T_{m}}+\ln \left(\frac{E_{d e s}}{R k_{0} n \theta^{n-1}}\right)
$$

where a plot of $\ln \left(T_{m}^{2} / \phi\right)$ vs $1 / T_{m}$ has slope $E_{d e s} / R$ and intercept $\ln \left(E_{d e s} /\left(R k_{0}\right)\right)$. You will note that this relationship does not require that the reaction order be known to 
determine $E_{A}$. For example, for a second order reaction $n=2$ as proposed here, a plot $\ln \left(\phi / T_{m}^{2}\right)$ vs $1 / T_{m}$ has slope $-E_{d e s} / R$. However, to determine the pre-exponential $\left(k_{o}\right)$ from the intercept, $\theta$ and $\mathrm{n}$ must be known.

Figure 9 shows the influence of ramp rate on peak position (not all points are shown for clarity). As seen in this figure, as ramp rate was increased the location of the peak temperature increased. In general, the curves fit the relationship $\mathrm{T}_{\mathrm{m}}=\mathrm{r} \square \mathrm{x}$ the values of the constants $\mathrm{r}$ and $\mathrm{x}$ are presented in Table 2. However, some deviation from this relationship was for Peaks 4 and 5 at a ramp rate of $5.4 \mathrm{~K} / \mathrm{min}$. This deviation likely owes to our inability to properly fit peak positions at this ramp rate and not a deviation from the exponential relationship for $\mathrm{T}_{\mathrm{m}}$. Using the relationships in Table 2 to generate ideal functions, a Kissinger style plot of $\ln (\phi /$ $T_{m}{ }^{2}$ ) vs $1 / T_{m}$ is presented in Figure 10 , the slope corresponding to $E_{\text {des. }}$. The calculated $\mathrm{E}_{\mathrm{des}}$ for each peak is also presented in Table 2. Literature values for the activation energy for $\mathrm{UH}_{3}$ powder decomposition range from $40 \mathrm{KJ} /$ mole (Stakebake [27]) to over $70 \mathrm{KJ} / \mathrm{mole}$ (Lindner $79 \mathrm{~kJ} / \mathrm{mole}$ [28], Condon $72 \mathrm{~kJ} / \mathrm{mole}$ [29]). With respect to the values of the activation energy calculated for the $\mathrm{UH}_{3}$ peaks, Peak 1- 3, we find values between $15-27 \mathrm{~kJ} /$ mole (Table 2), below the literature range for powders. In our previous study on UH3 powder using TDS we calculated a $\mathrm{E}_{\text {des }}$ of 43 $\mathrm{kJ} / \mathrm{mol}$ indicating that this lower value is intrinsic to metal coupons. Contrary to the general observation that lower temperature peaks have lower activation energies and the simple concept that increasing temperature increases the kinetic energy of the system it is seen in Table 2 for the $\mathrm{UH}_{3}$ Peaks that $\left.\left(\mathrm{E}_{\mathrm{des}}\right)_{3}<\mathrm{E}_{\mathrm{des}}\right)_{2}<\left(\mathrm{E}_{\mathrm{des}}\right)_{1 . .}$ 
However, similar relationships between activation energy and temperature have been observed by Yang [30] as well as Borchers [31] for desorption from titanium hydride. In those works, three TDS peaks were observed in the transition from the hydride to Ti metal and were attributed to the $\varepsilon \mathrm{TiH}_{2}(743 \mathrm{~K}), \delta \mathrm{TiH}_{2-\mathrm{x}}(817 \mathrm{~K})$ and $\gamma \mathrm{TIH}(920 \mathrm{~K})$ phases.[31] With respect to activation energies, the transition at $817 \mathrm{~K}$ had the highest activation energy. Therefore, either the Kissinger / Redhead analysis must be invalid for these hydride systems or there must be another explanation for this observation such as secondary trapping. The later is supported by two observations, 1) the reaction is second order as described above and 2) the trend of decreasing activation energy with increasing peak temperature for Peaks 13 is related to the area under the peak, that is, the concentration of hydrogen desorbing as seen in Figure 11 that plots the area under the spectra for each of the fitted curves at a single ramp rate $(5 \mathrm{~K} / \mathrm{min}$.). As seen in this figure, even for the same peak (same symbol) the temperature associated with $\mathrm{T}_{\mathrm{m}}$ increased with increasing hydrogen desorbed. To help quantify this relationship, we turn to the work by Elovich [32] on the adsorption of CO surfaces, and later by Taylor [33] and then Low [34] for the adsorption of hydrogen, which found that the surface coverage of adsorbed species with time was non-linear, specifically:

$$
d \theta / d t=a e^{-\alpha \theta}
$$

where a and $\alpha$ are constants. With respect to the Elovich study, the equation describes the exponential decrease in the rate of adsorption of $\mathrm{CO}$ with the increase in the fractional of coverage of CO. Thus, interactions between adsorbates are concentration dependent and can play an important role in kinetics. Since the 
original Elovich study, Eq. 4 has been frequently used in thermally programmed desorption (TPD) investigations of surface adsorbate [12]:

$$
d \theta / d t=k_{0} \theta^{n} \exp \left\{-\left(E_{d e s}-\alpha \theta\right) / R T\right\}
$$

This expression can be used to evaluate either adsorbate interactions that increase the activation energy (negative $\alpha$ ) or adsorbate interactions that decrease the activation energy (positive $\alpha$ ). With respect to the TDS data reported here and the trend in Figure 11, it is proposed that hydrogen-hydrogen interactions within the lattice result in a decrease in the measured activation energy. For example, in Figure 11 the Pre-Peak and Peaks 1-3 have a disproportionately low concentration of hydrogen as compared to Peaks 4-6. Correspondingly their desorption energy is relatively high. In contrast, there is a net attraction at high concentrations seen in Peak 4-6 that decreases the desorption energy associated with these peaks.

To evaluate the extent of these interactions, $\alpha$ in Eq. 5 must first be determined. This correction value is determined by first re-arranging Eq 5 then integrating. The resulting expression has the form:

$$
\theta=-\frac{R T}{\alpha} \ln \left(t-t_{0}\right)+\frac{a}{\alpha}
$$

Thus, a plot of $\theta$ vs $\ln \left(\mathrm{t}-\mathrm{t}_{0}\right)$ has slope $-\mathrm{RT} / \alpha$ and intercept $\mathrm{a} / \alpha$. We have done this for Peaks 1-3 (plots not shown) and found that the resulting correction is on the order of $-1.9 \mathrm{~kJ} / \mathrm{mol}$ (Peak 1) to $-2.2 \mathrm{~kJ} / \mathrm{mol}$ (Peak 3) as seen in Table 2. This is the net decrease in activation energy associate with each peak due hydrogen-hydrogen interactions. To determine the true desorption energy for each peak, this value 
would need to be added to value determined from the Kissinger analysis in Table 2 . Because there is no concentration dependence of $E_{\text {des }}$ for Peaks 4-6, one might assume that the effect saturates and a value of $-2.2 \mathrm{~kJ} / \mathrm{mol}$ describes the interaction energy for these peaks. Therefore, we conclude that there are positive hydrogenhydrogen interactions within the $\alpha$-U lattice that result in 1 ) second order reaction kinetics and 2) the under estimation of the desorption energy measured during TDS experiments.

\section{Conclusions}

From the results of the TDS data for the desorption of hydrogen from $\alpha$-U it can be concluded that:

- Assuming second order reaction kinetics and Gaussian shaped peaks, deconvolution to the TDS spectrum for $\mathrm{H}$ in $\alpha$-U finds six distinct peaks below the $\alpha$ to $\beta$ phase transition at $935 \mathrm{~K}$. While we do not believe the number of peaks would have changed if we had assumed first order reaction kinetics and corresponding sigmoidal curves, differences in peak area and peak position $\left(\mathrm{T}_{\mathrm{m}}\right)$ would no doubt be observed.

- The first 3 peaks in the spectra for hydrogen desorption in $\alpha$-U were associated with the transformation of $\mathrm{UH}_{3}$. This was concluded based on comparison of the polycrystalline spectra with a $\mathrm{UH}_{3}$ standard. As the galvanostatic process used here to charge the samples with hydrogen was optimized to avoid $\mathrm{UH}_{3}$ formation and no $\mathrm{UH}_{3}$ pits were formed on the surface, it is believed a thin surface layer of non- 
stoichiometric $\mathrm{UH}_{3}$ was formed during charging. This surface layer had a tan translucent color.

- One of the $\mathrm{UH}_{3}$ peaks was also associated with dislocations or deformation twins in the material. This conclusion was made after comparison of annealed $\alpha$-U samples with plastically deformed samples. Further, the phase transformation process does not alter the increased hydrogen adsorption at these sites consistent with work elsewhere on palladium hydride.

- The remaining peaks in the TDS spectra were associated with inclusions and high angle boundaries (e.g. grain boundaries). This conclusion was based on comparison of polycrystalline $\alpha$-U samples with high purity single crystal $\alpha-U$.

- The desorption energy $\left(E_{\text {des }}\right)$ calculated for each trap site using the Kissinger style analysis was consistent with the range of desorption energies for hydrogen from $\mathrm{UH}_{3}$, however, contrary to the general finding in the literature that lower temperature peaks have lower activation energies, the low temperature $\mathrm{UH}_{3}$ peaks had the highest desorption energies. It was further concluded that this trend was related to the concentration of hydrogen desorbing with peaks associated with larger amounts of desorbing hydrogen having lower activation energies as quantified using the method proposed by Elovich. This positive hydrogen-hydrogen interaction within the $\alpha$-U lattice resulted in 1) second order reaction kinetics and 2) the under estimation of the desorption energy measured during TDS experiments.

\section{Acknowledgements}


Experimental work for this paper was performed at the Los Alamos National Laboratory under the Enhanced Surveillance Campaign, Thomas Zocco program manager. Data analysis and manuscript preparation were supported by the University of Akron. LANL is operated by Los Alamos National Security LLC for the National Nuclear Security Administration of the U.S. Department of Energy under contract DE-AC52-06NA25396. The authors thank Marilyn Hawley for help with the STM measurements and Michael Baskes and Mary Ann Hill for insightful discussions. The uranium single crystal used in this work was prepared by CMT Division, Argonne National Laboratory.

\section{References}

[1] J. Bloch, F. Simca, M. Kroup, A. Stern, D. Shmariahu, M. Mintz, J. Less-Common Met. 103 (1984) 163.

[2] J. Bloch, J. Alloys Compd. 361 (2003) 130.

[3] J.R. Kirkpatrlck, J. Chem. Phys. 85 (1981) 3444.

[4] T.C. Totemeier, J. Nucl. Mater. 278 (2000) 301.

[5] A. Loui, The Hydrogen Corrosion of Uranium $\square$ : Identification of Underlying Causes and Proposed Mitigation Strategies, Lawrence Livermore National Lab \#LLNL-TR-607653, Livermore, 2012.

[6] C. Taylor, R. Lillard, Acta Mater. 57 (2009) 4707.

[7] C. Taylor, T. Lookman, R. Lillard, Acta Mater. 58 (2010) 1045.

[8] G.L. Powell, J.B. Condon, Anal. Chem. 45 (1973) 2349.

[9] R.S. Lillard, C.D. Taylor, J.R. Wermer, N.A. Mara, J.C. Cooley, J. Nucl. Mater. 444 (2014) 49. 
[10] R. Griffiths, J.A. Pryde, Trans. Faraday Soc. 64 (1968) 507.

[11] T. Flanagan, in:, A. Andresen, A. Maeland (Eds.), Hydrides Energy Convers., William Cloves \& Sons, Lmtd., London, 1978, pp. 135-150.

[12] R.I. Masel, Principles of Adsorptionand Reaction on Solid Surfaces, John Wiley \& Sons, In., New York, 1996.

[13] H.E. Kissinger, Anal. Chem. 29 (1957) 1702.

[14] P.A. Redhead, Vascuum 13 (1963) 253.

[15] J. Lashley, B. Lang, J. Boerio-Goates, B. Woodfield, G. Schmiedeshoff, E. Gay, C. McPheeters, D. Thoma, W. Hults, J. Cooley, R. Hanrahan, J. Smith, Phys. Rev. B 63 (2001) 224510.

[16] R.D. Kolasinski, A.D. Shugard, C.R. Tewell, D.F. Cowgill, Uranium for Hydrogen Storage Applications $\square$ : A Materials Science Perspective, Sandia National Lab \# SAND2010-5195, Albuquerque, 2010.

[17] G.L. Powell, W.L. Harper, J. Less-Common Met. 172-174 (1991) 116.

[18] M. Salloum, P.E. Gharagozloo, Chem. Eng. Sci. 116 (2014) 452.

[19] J.J. Katz, E. Rabinowitch, The Chemistry of Uranium, Chapter 8, McGraw-Hill, New York, 1951.

[20] C.F. St. John, Deformation of Alpha-Uranium, The University of British Columbia, 1962

[21] T.B. Flanagan, J.D. Clewley, J.F. Lynch, J. Less-Common Met. 41 (1975) 343.

[22] R. Arkush, A. Venkert, M. Aizenshtein, S. Zalkind, D. Moreno, M. Brill, M. MIntz, N. Shamir, J. Alloys Compd. 244 (1996).

[23] S.W. Smith, J.R. Scully, Metall. Meterials Trans. A 31 (2000) 179.

[24] T.B. Scott, G.C. Allen, I. Findlay, J. Glascott, Philos. Mag. 87 (2007) 177.

[25] G. Crocker, J. Nucl. Mater. 16 (1965) 306.

[26] M.A. Hill, R.K. Schulze, J.F. Bingert, R.D. Field, R.J. McCabe, P.A. Papin, J. Nucl. Mater. 442 (2013) 106.

[27] J.L. Stakebake, J. Electrochem. Soc. 126 (1979) 1596. 
[28] D.L. Lindner, J. Less-Common Met. 157 (1990) 139.

[29] J.B. Condon, E.A. Larson, J. Chem. Phys. 59 (1973) 855.

[30] D. Yang, Sci. China Ser. B 47 (2004) 512.

[31] C. Borchers, T.I. Khomenko, a. V. Leonov, O.S. Morozova, Thermochim. Acta 493 (2009) 80.

[32] S.Y. Elovich, G.R. Zhabrova, Russ. J. Phys. Chem. 13 (1939) 1761.

[33] H.A. Taylor, N. Thon, J. Am. Chem. Soc. 74 (1952) 4169.

[34] M.J.D. Low, J. Phys. Chem. 65 (1961) 887. 
Table 1 - Comparison of peak positions (in $\mathrm{K}$ ) at $5.4 \mathrm{~K} / \mathrm{min}$ for the three different types of samples analyzed. Data are also compared with position of peaks from pure $\mathrm{UH}_{3}$ powder.[9]

\begin{tabular}{lllllll}
\hline & 1 & 2 & 3 & 4 & 5 & 6 \\
\hline hydride[ref] & 518 & 547 & 585 & - & - & - \\
poly & 521 & 556 & 607 & 681 & 793 & 905 \\
& $+/-15$ & $+/-15$ & $+/-8$ & $+/-19$ & $+/-11$ & $+/-7$ \\
\multirow{2}{*}{ deformed } & 496 & 532 & 607 & 663 & 770 & 897 \\
& $+/-11$ & $+/-16$ & $+/-4$ & $+/-14$ & $+/-10$ & $+/-9$ \\
\multirow{2}{*}{ single } & 532 & 574 & 631 & 700 & 778 & 891 \\
& $+/-15$ & $+/-6$ & $+/-16$ & $+/-24$ & $+/-16$ & $+/-9$ \\
\hline
\end{tabular}

Table 2 - Values from the curve fits of the data in Figure 8 to the relationship $\mathrm{T}_{\mathrm{m}}=\mathrm{r} \square \mathrm{x}$ for each peak $\left(\mathrm{T}_{\mathrm{m}}\right)$. Corresponding $\mathrm{E}_{\text {des }}$ from the slope of the curves in Figure 9 and Eq. 3. as well as the adjusted value assuming hydrogen-hydrogen interactions within the uranium lattice.

\begin{tabular}{|c|c|c|c|c|c|c|}
\hline & 1 & 2 & 3 & 4 & 5 & 6 \\
\hline$r(k J)$ & 422.6 & 428.5 & 428.7 & 448.0 & 519.4 & 625.5 \\
\hline $\mathrm{x}$ & 0.1161 & 0.1442 & 0.1813 & 0.2108 & 0.2015 & 0.1851 \\
\hline $\begin{array}{l}E_{\text {des }} \text { Kissinger } \\
(\mathrm{kJ} / \text { mole })\end{array}$ & 26.5 & 20.6 & 15.3 & 12.9 & 16.0 & 21.7 \\
\hline trap ID & $\begin{array}{l}\mathrm{UH}_{3} / \\
\text { dislocations }\end{array}$ & $\mathrm{UH}_{3}$ & $\mathrm{UH}_{3}$ & twins & inclusions & $\begin{array}{l}\text { grain } \\
\text { boundaries }\end{array}$ \\
\hline$\alpha \theta(\mathrm{J} / \mathrm{mole})$ & -1883 & -2009 & -2194 & - & - & - \\
\hline $\begin{array}{l}\mathrm{E}_{\text {des }} \text { adjusted } \\
(\mathrm{kJ} / \mathrm{mole})\end{array}$ & 28.4 & 22.6 & 17.5 & & & \\
\hline
\end{tabular}




\section{Figure List}

Figure 1 Energy diagram for hydrogen in a material depicting the various boundaries where: $E_{D}$ is the energy associated with diffusion, $E_{A}$ is the binding energy associated with traps, and $E_{\text {des }}$ is the measured energy of desorption equal to the sum of $\mathrm{E}_{\mathrm{D}}+\mathrm{E}_{\mathrm{A}}$.

Figure 2 Experimental TDS from polycrystalline $\alpha$-U showing at a ramp rate of 5 $\mathrm{K} / \mathrm{min}$ a) RGA current at mass 2 vs time and b) current vs temperature data as well as the corresponding Gaussian fit to the curve.

Figure 3 a) Comparison of TDS spectra for $\alpha-U$ with $\mathrm{UH}_{3}$ powder data and b) corresponding curve fits to the data. The data were generated for a ramp rate of 5 $\mathrm{K} / \mathrm{min}$

Figure 4 a) Comparison of the TDS spectra for an annealed polycrystalline $\alpha-U$ sample with data from a sample that had been plastically deformed and b) the corresponding fit to the data. The data were generated for a ramp rate of $5 \mathrm{~K} / \mathrm{min}$.

Figure 5 a) SEM image of an inclusion in the polycrystalline material, b) energy dispersive spectra of the dark regions of the inclusion showing the presence of both oxygen and carbon and c) EDS spectra of the lighter regions of the inclusions were carbon rich.

Figure 6 Statistical distributions of a) inclusion area and b) equivalent circular diameter in the polycrystalline material.

Figure 7 a) Typical TDS data from single crystal $\alpha-U$ and b) the corresponding fit. The data were generated for a ramp rate of $5 \mathrm{~K} / \mathrm{min}$.

Figure 8 a) In situ EC-STM height map of a $\mathrm{U}^{238}$ sample in $2 \mathrm{wt} \%$ o-phosphoric acid $\left(\mathrm{E}=-690 \mathrm{mv}, \mathrm{E}_{\mathrm{T}}=-200 \mathrm{mV}, \mathrm{E}_{\mathrm{B}}=490 \mathrm{mV}, \mathrm{i}_{\mathrm{set}}=0.5 \mathrm{nA}\right)$ and $\left.\mathrm{b}\right)$ corresponding electrochemical potential between the STM tip and the sample surface for a constant tip.

Figure 9 The influence of ramp rate on peak position. Not all points are shown for clarity.

Figure 10 Kissinger style plot for determining the desorption energies associate with each peak.

Figure 11 A plot of $E_{\text {des }}(\square)$ and the area under the curve (proportional to hydrogen concentration) as a function of peak temperature $\left(\mathrm{T}_{\mathrm{m}}\right)$. The plots show an inverse relationship between activation energy and desorbed hydrogen for the hydride peaks. 


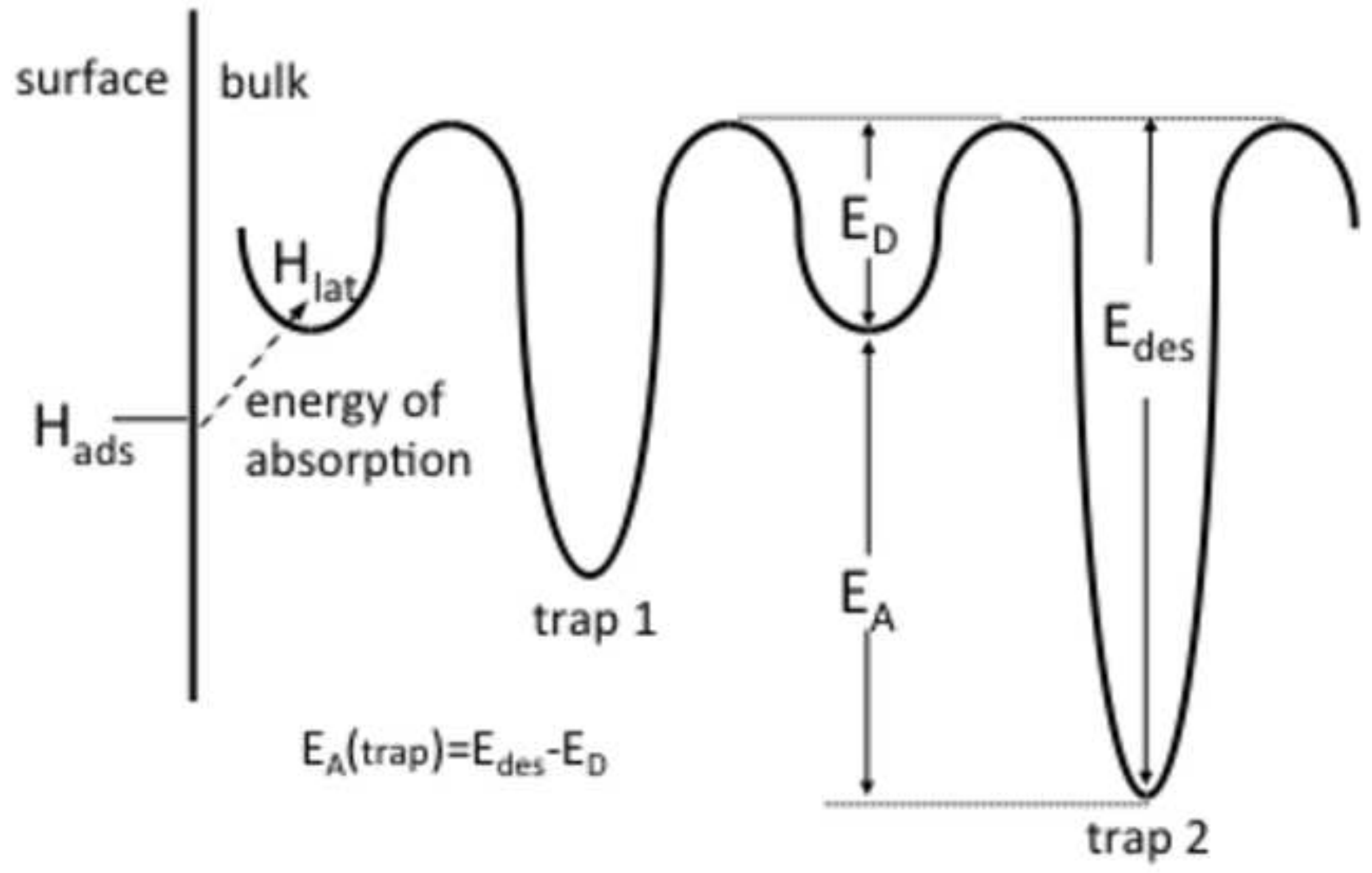




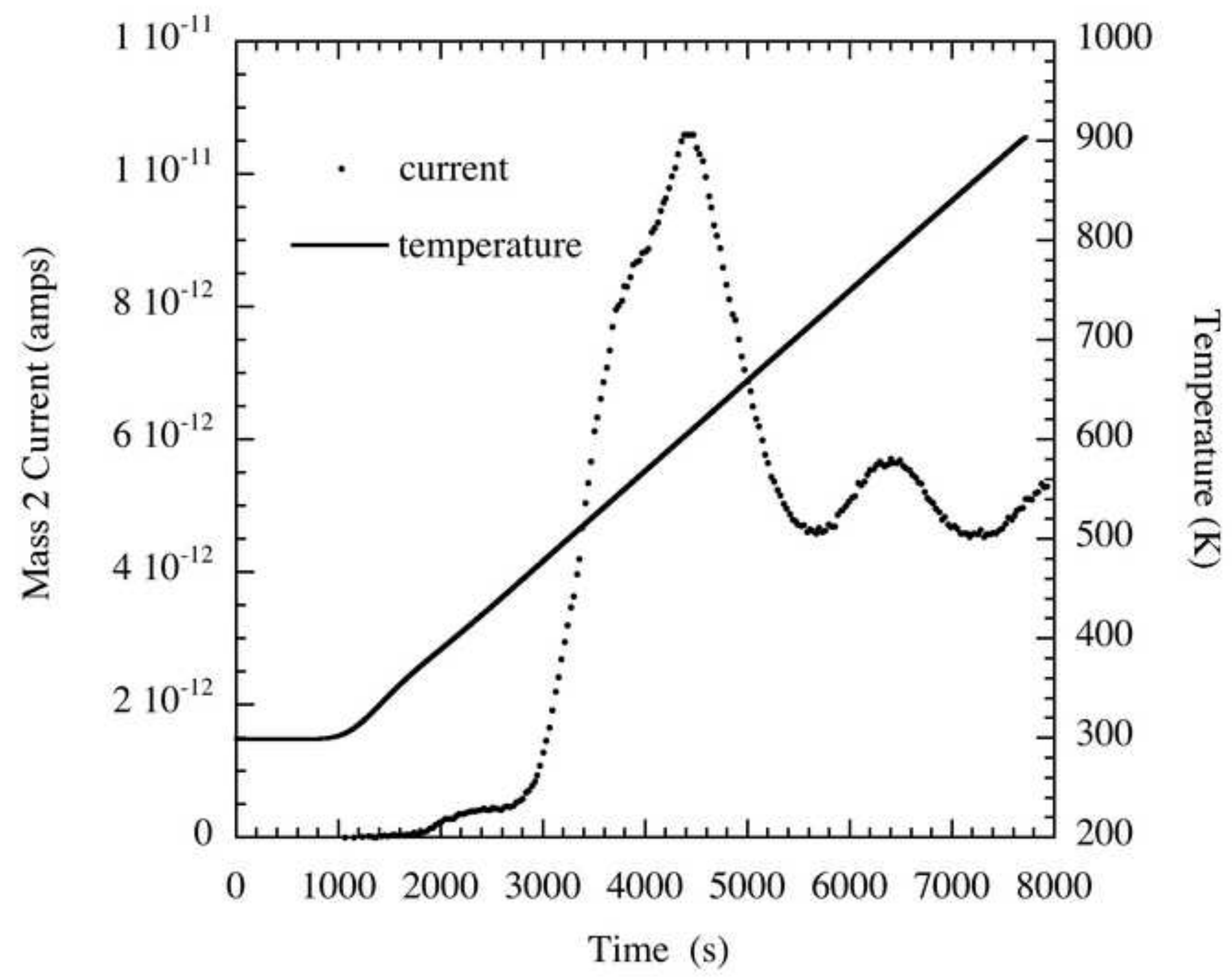

Time (s) 


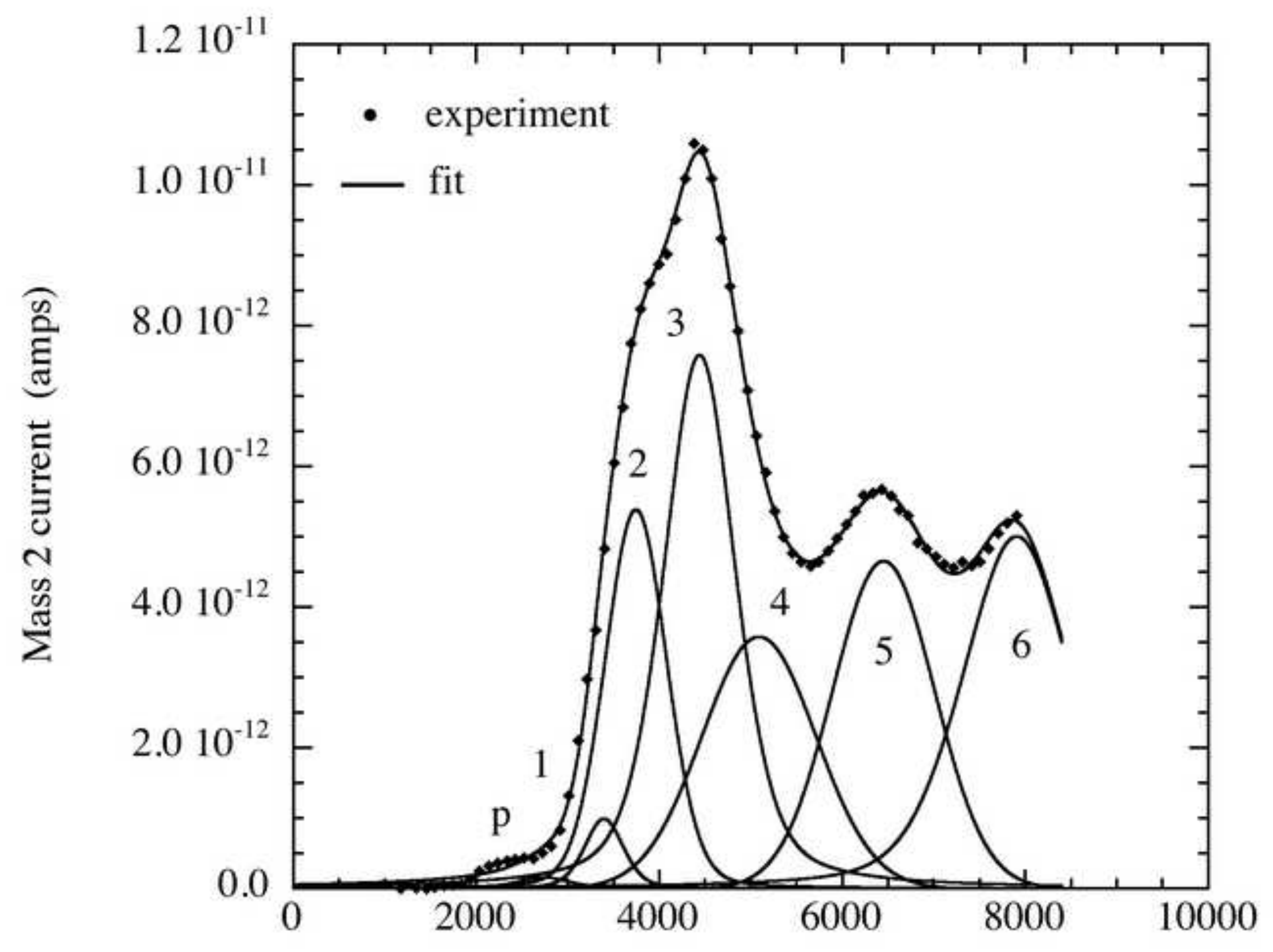

Time (s) 


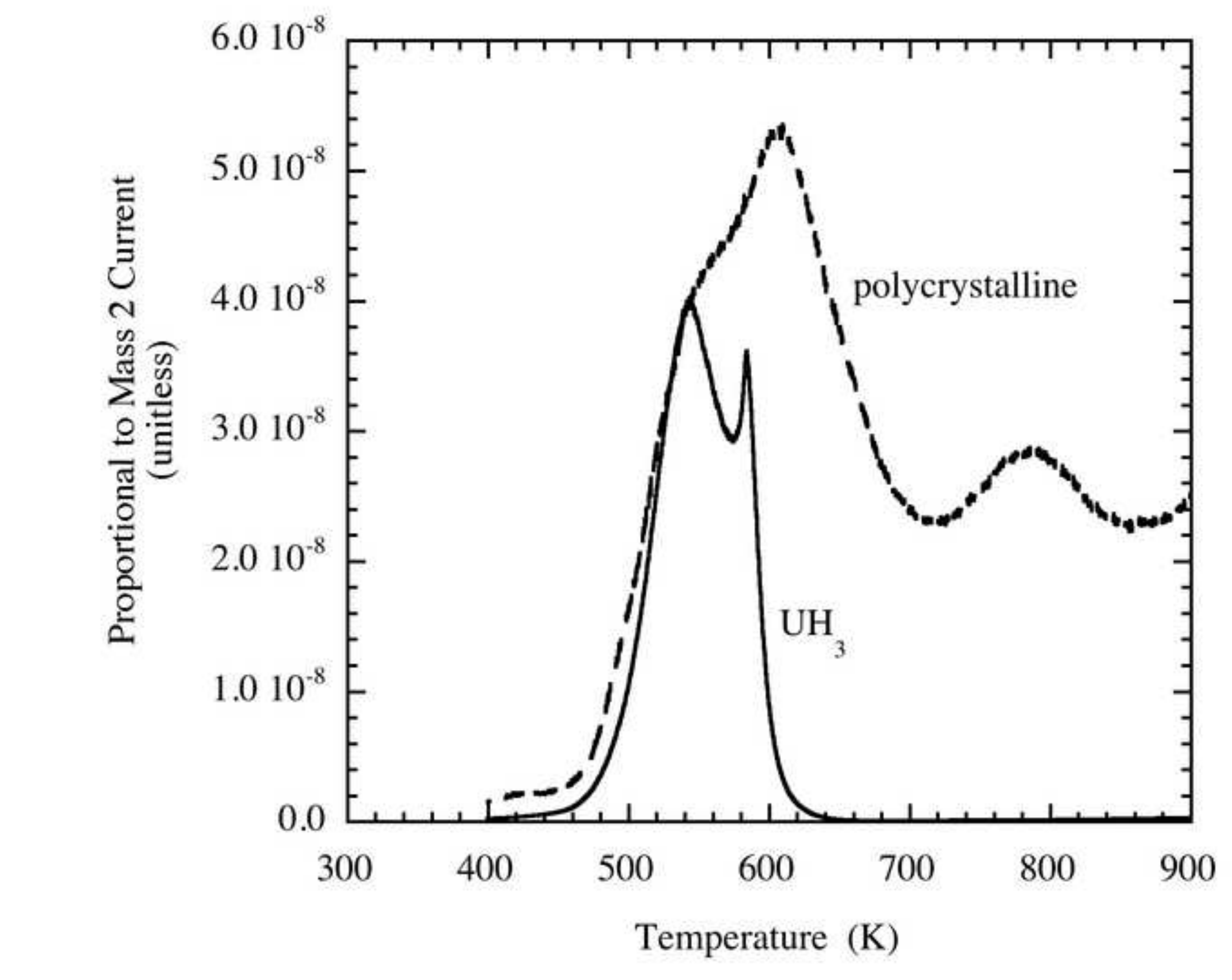




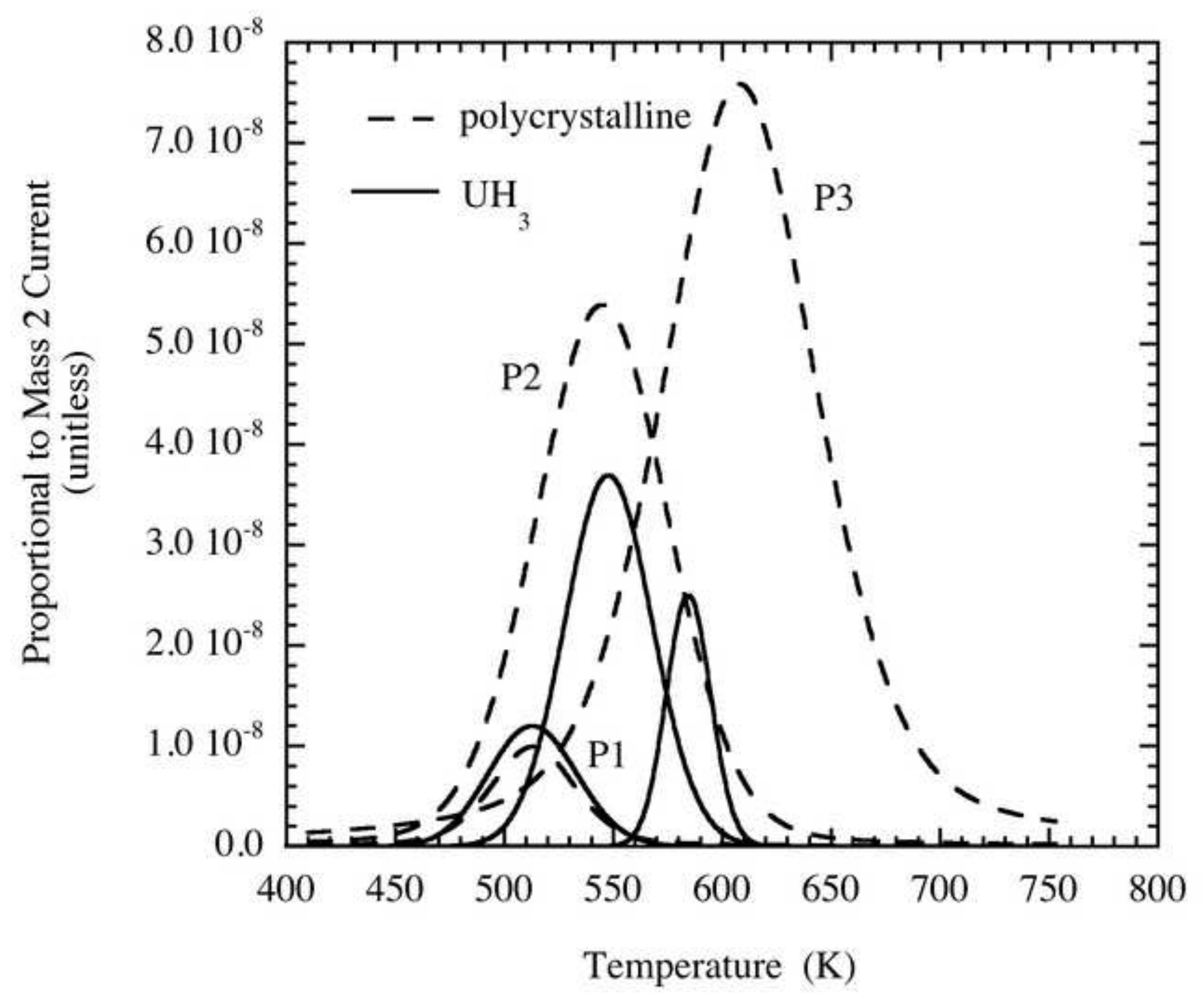




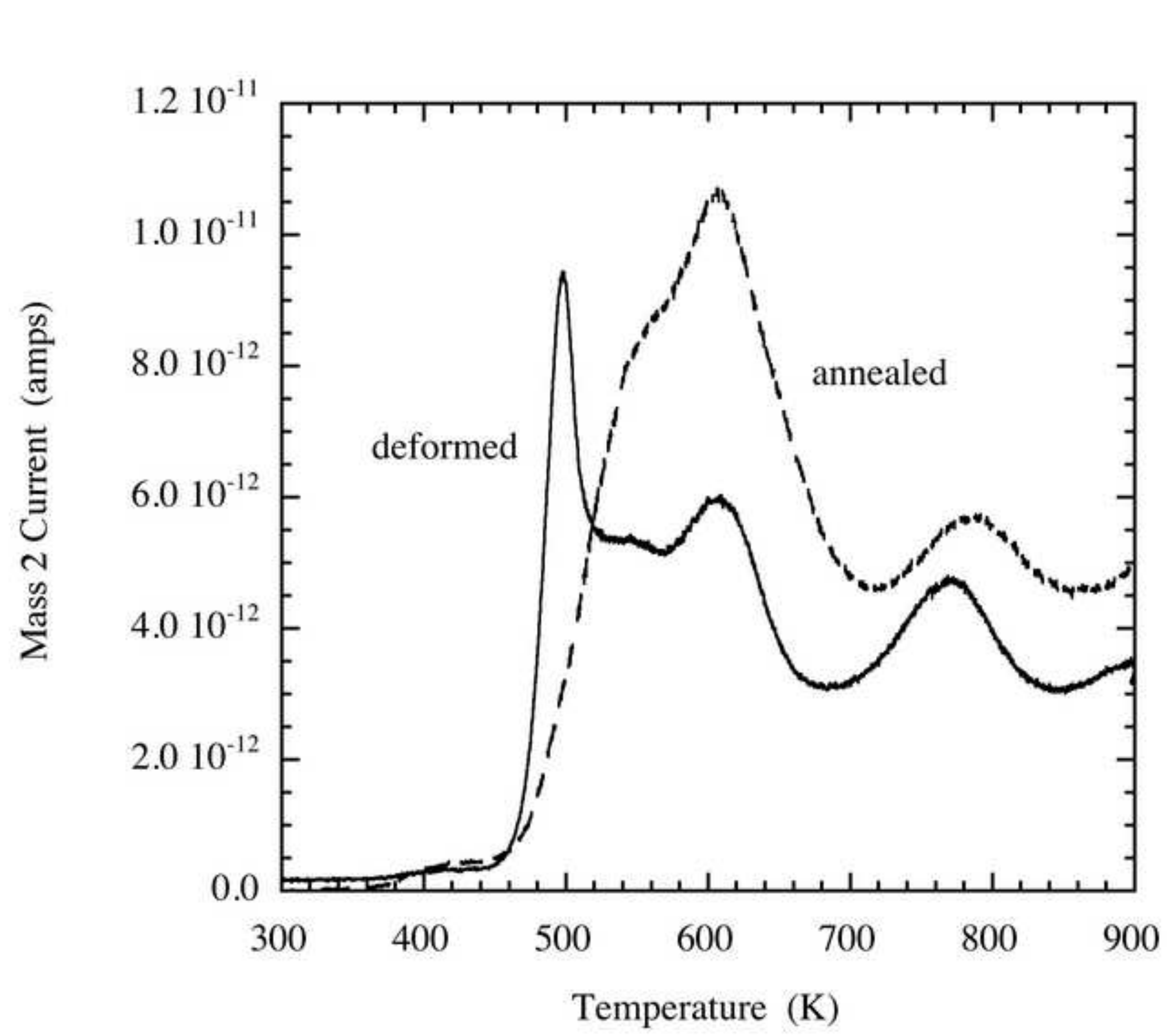




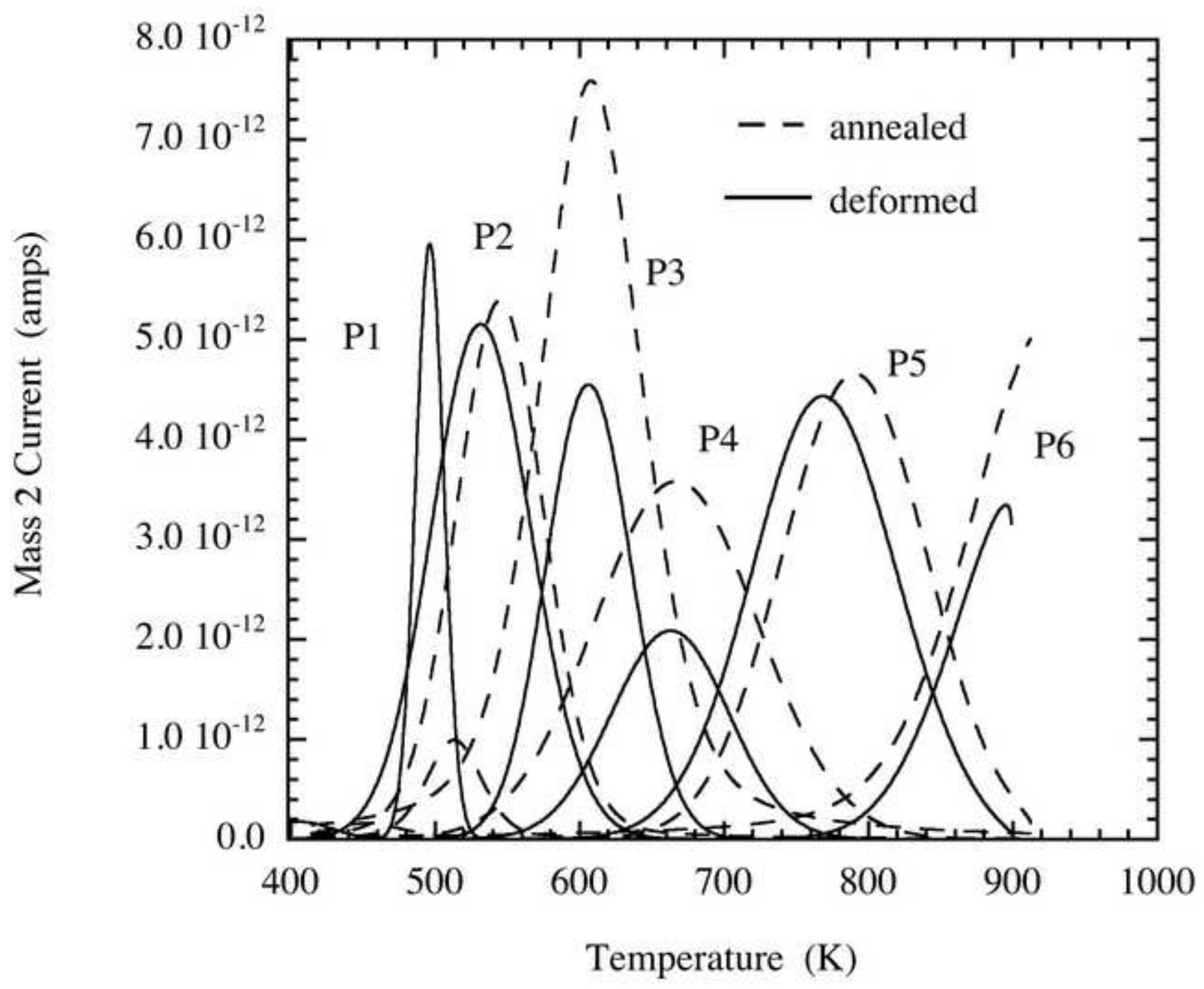




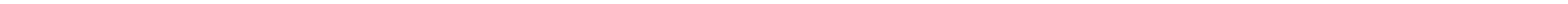

Figure $5 a$

\section{$5 \mu \mathrm{m}$}

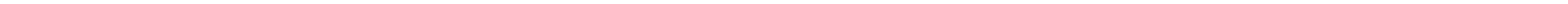

\section{Figure}
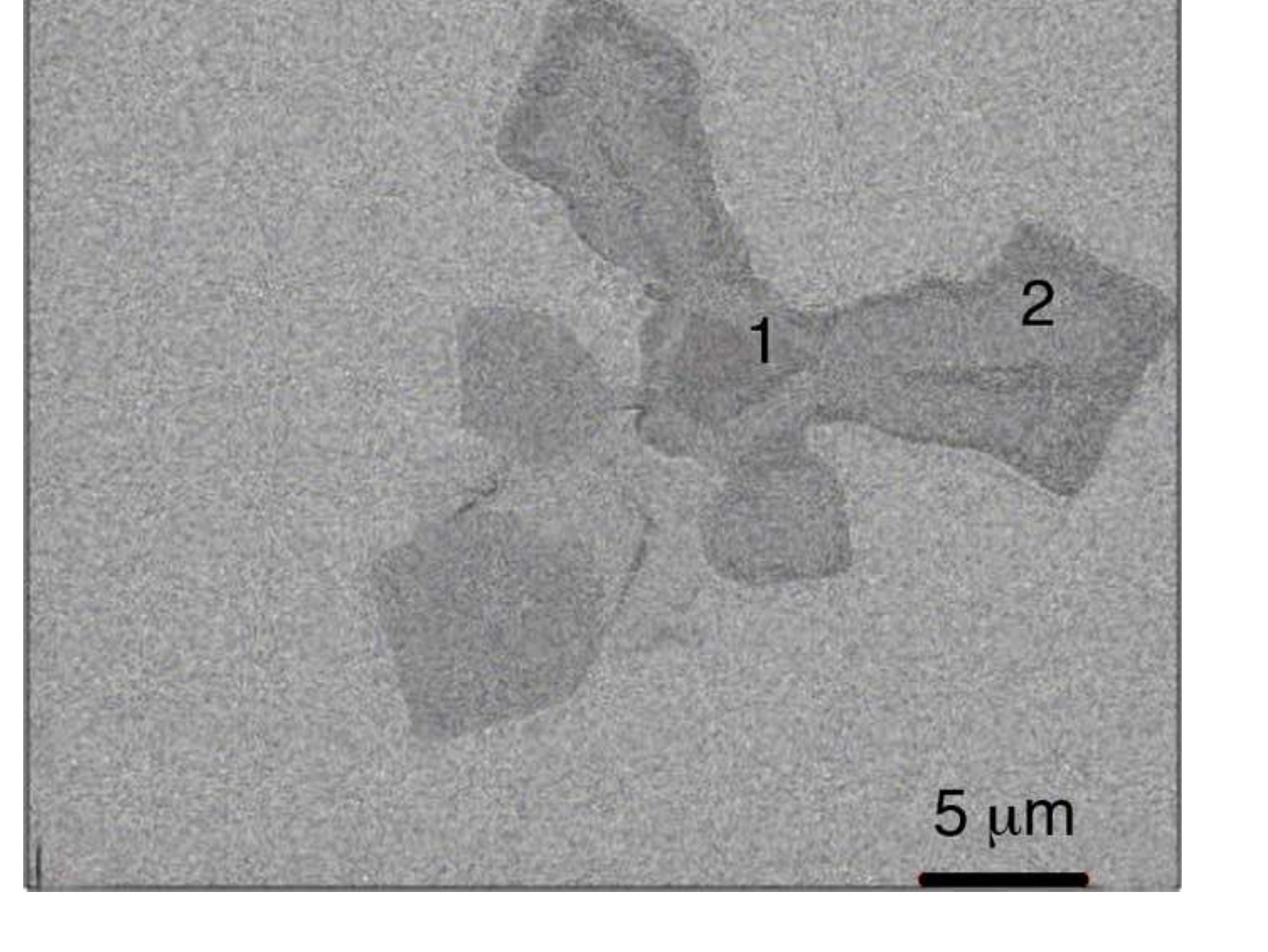


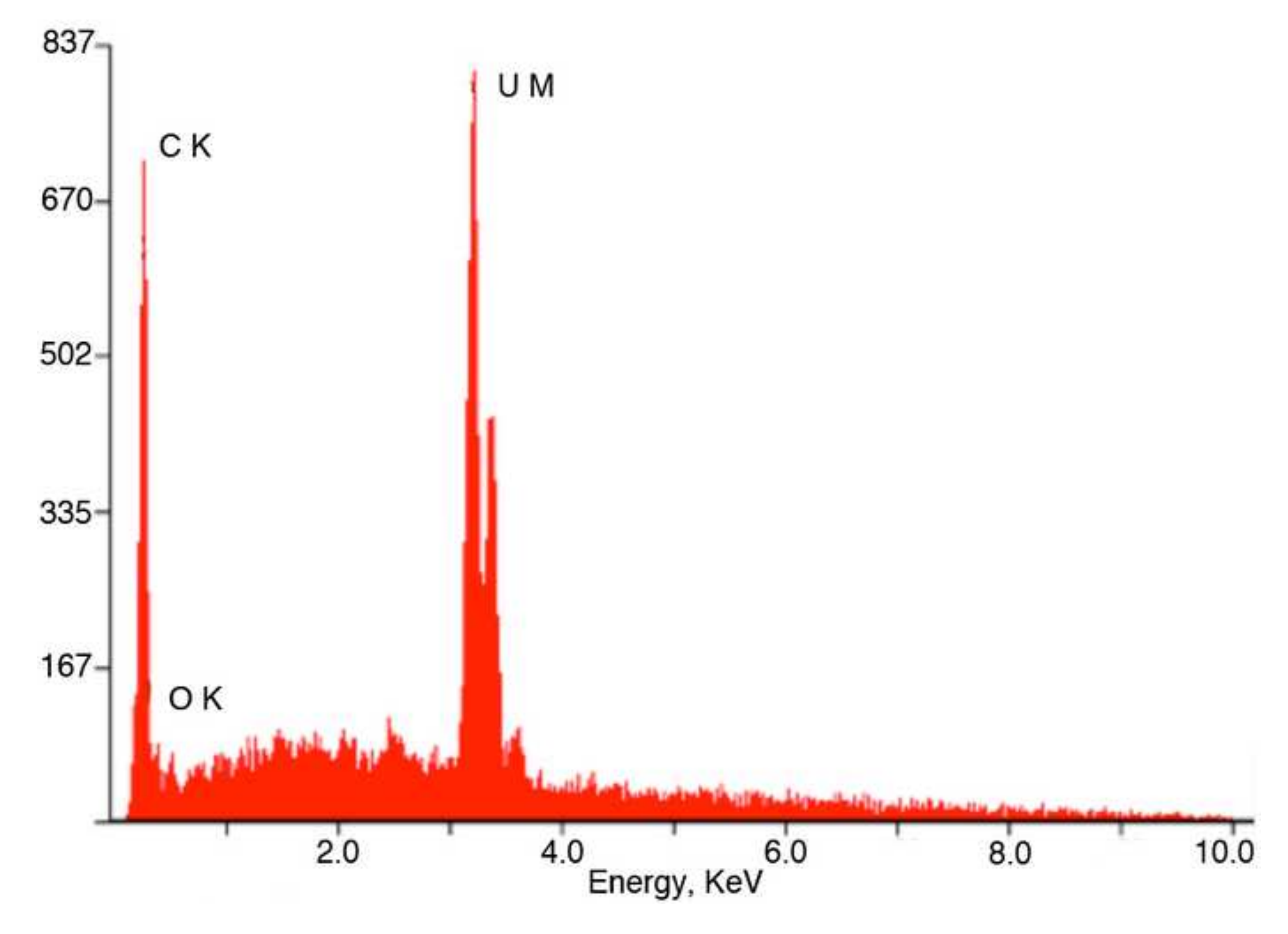




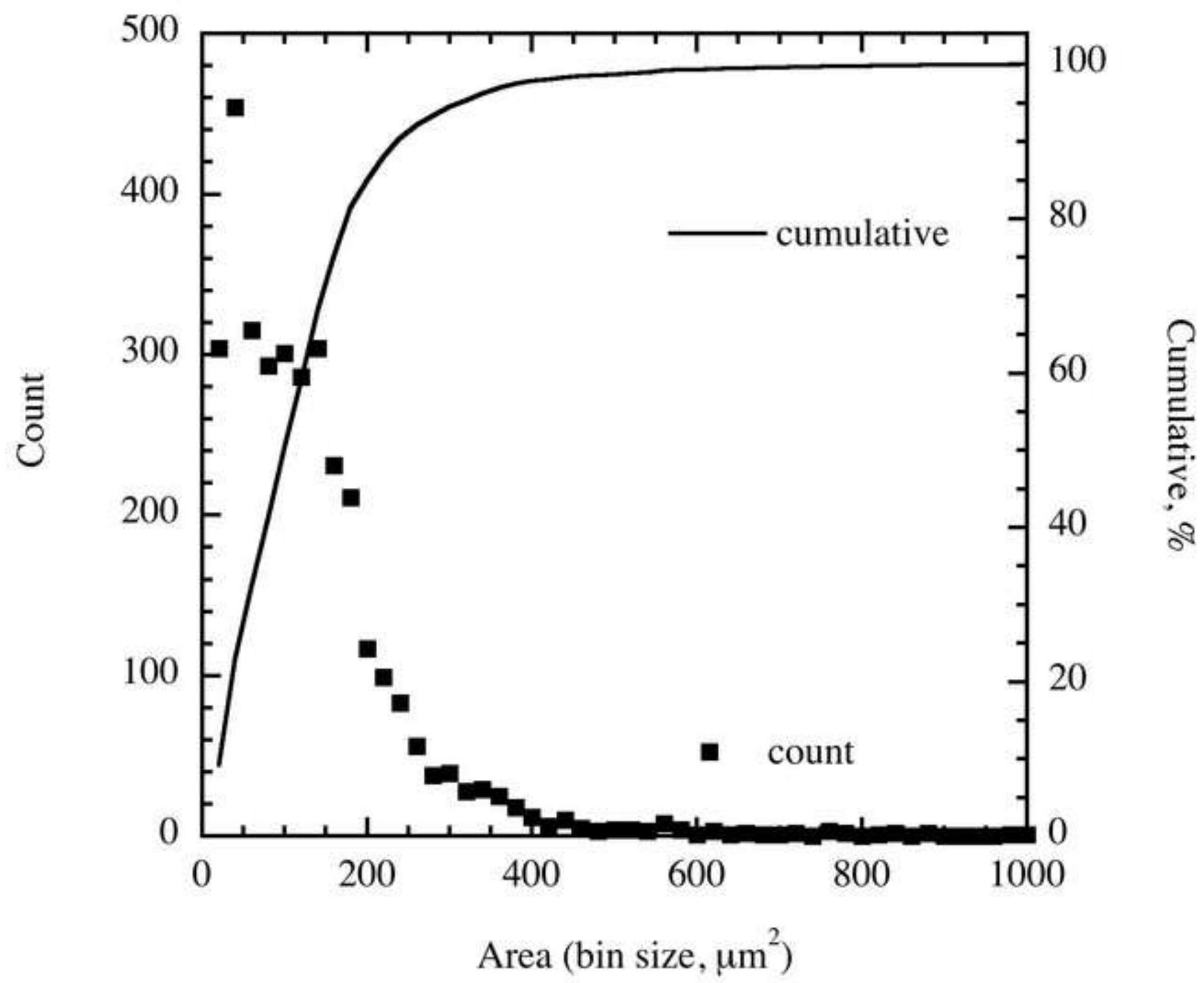




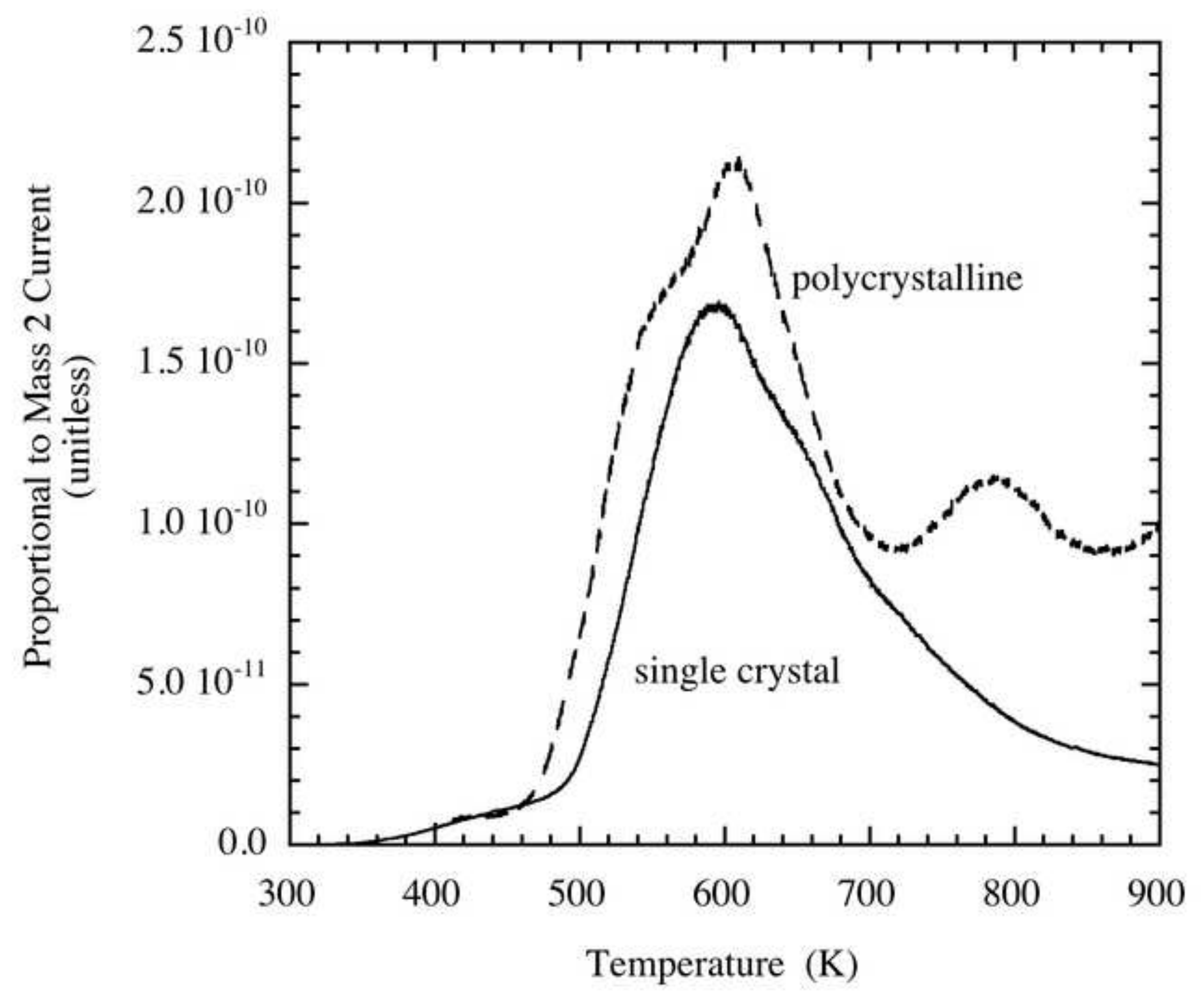




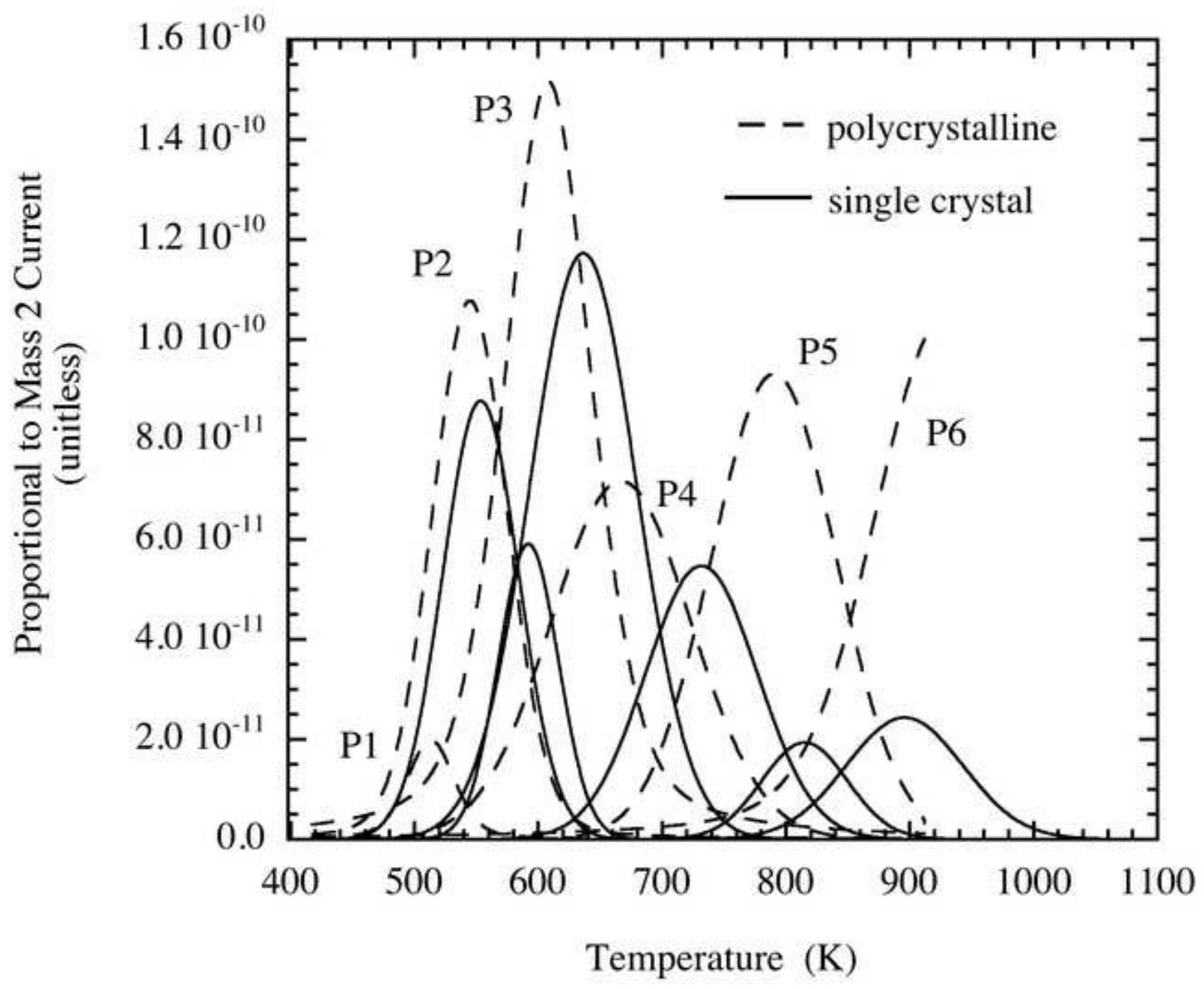




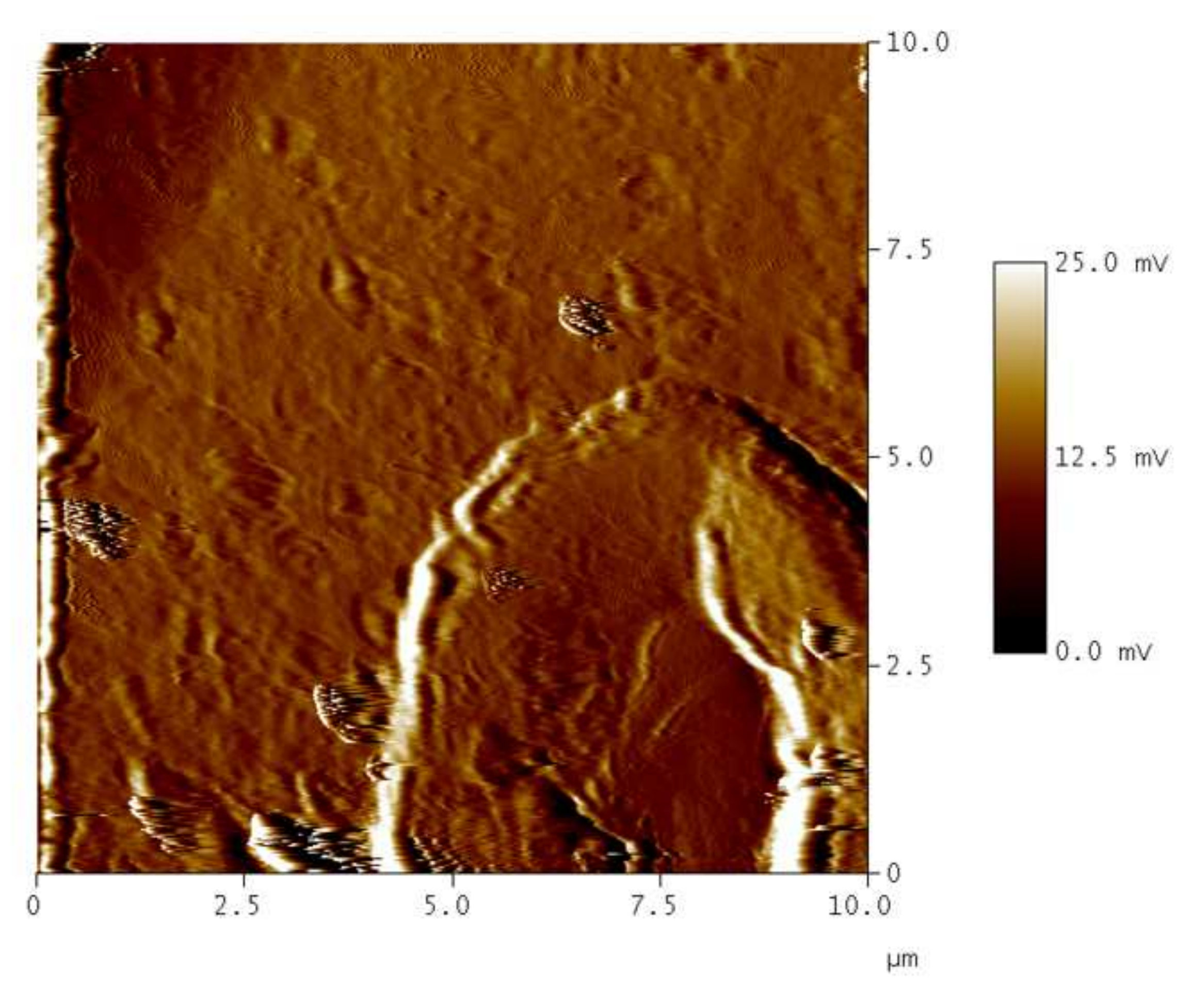

.

(3)
.

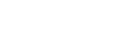

(6.
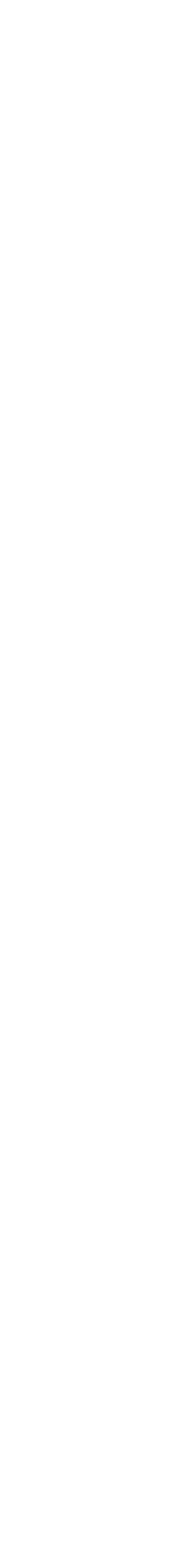


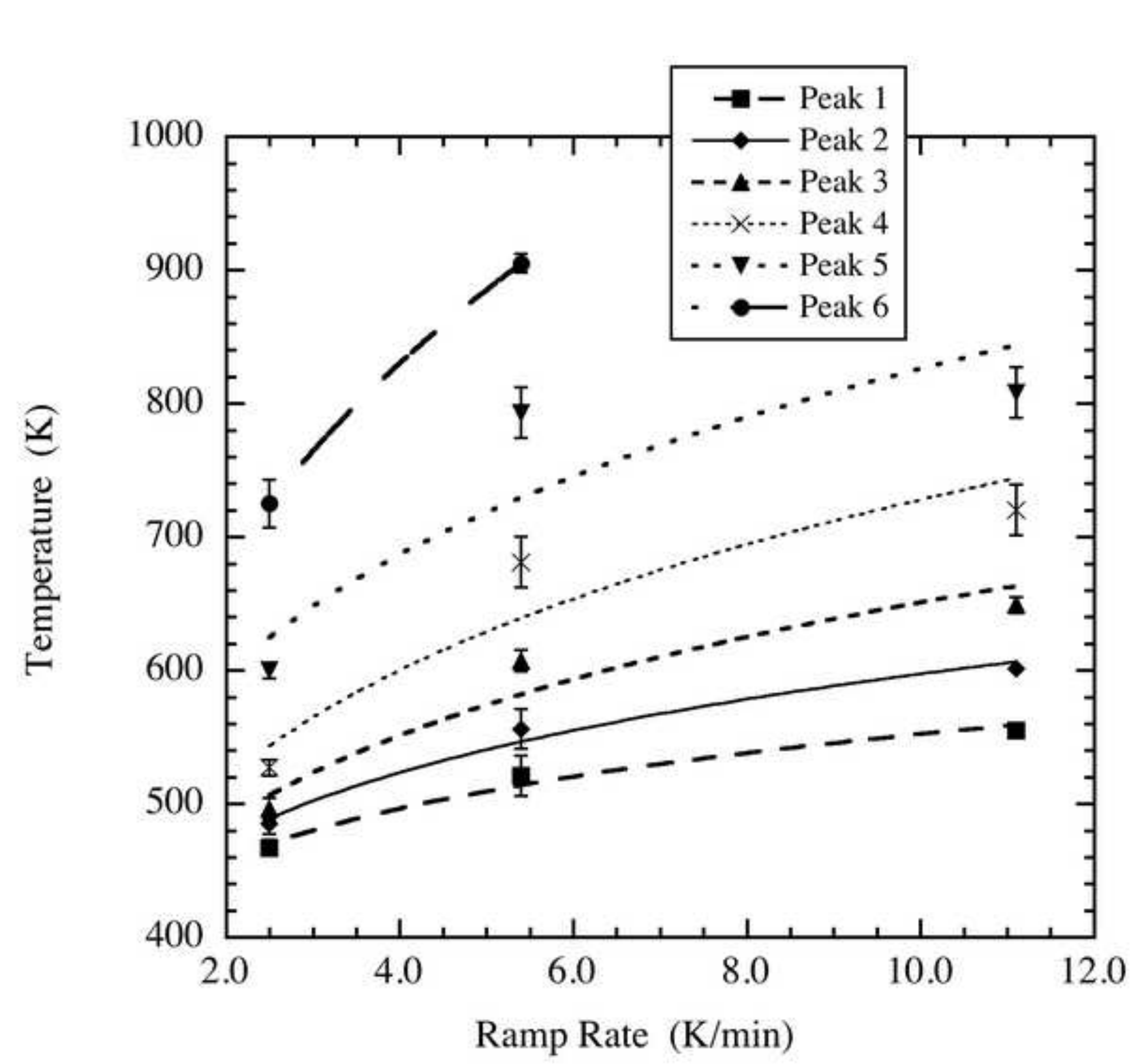




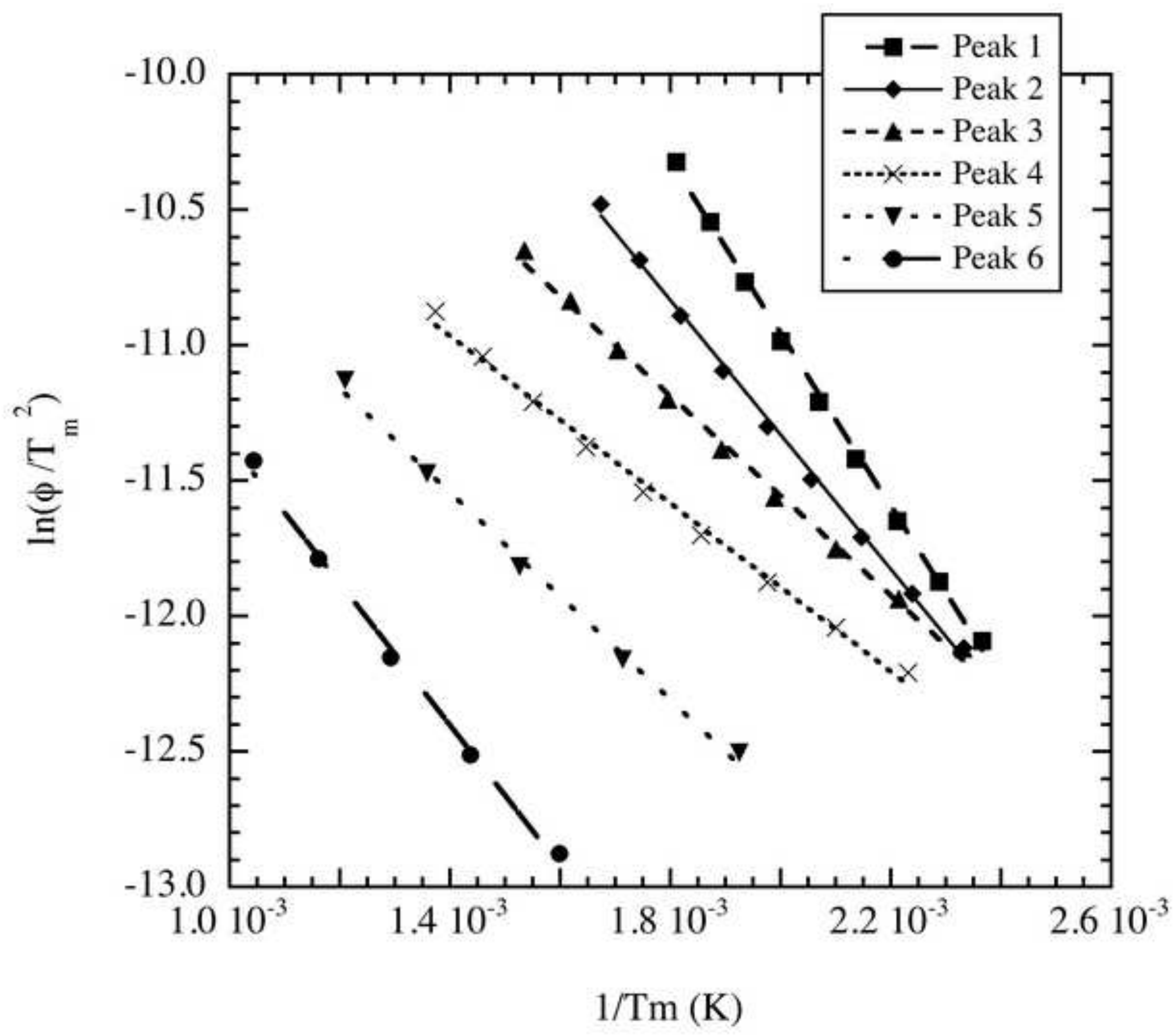


ㅁ Ea $\mathrm{kJ} / \mathrm{mole}$

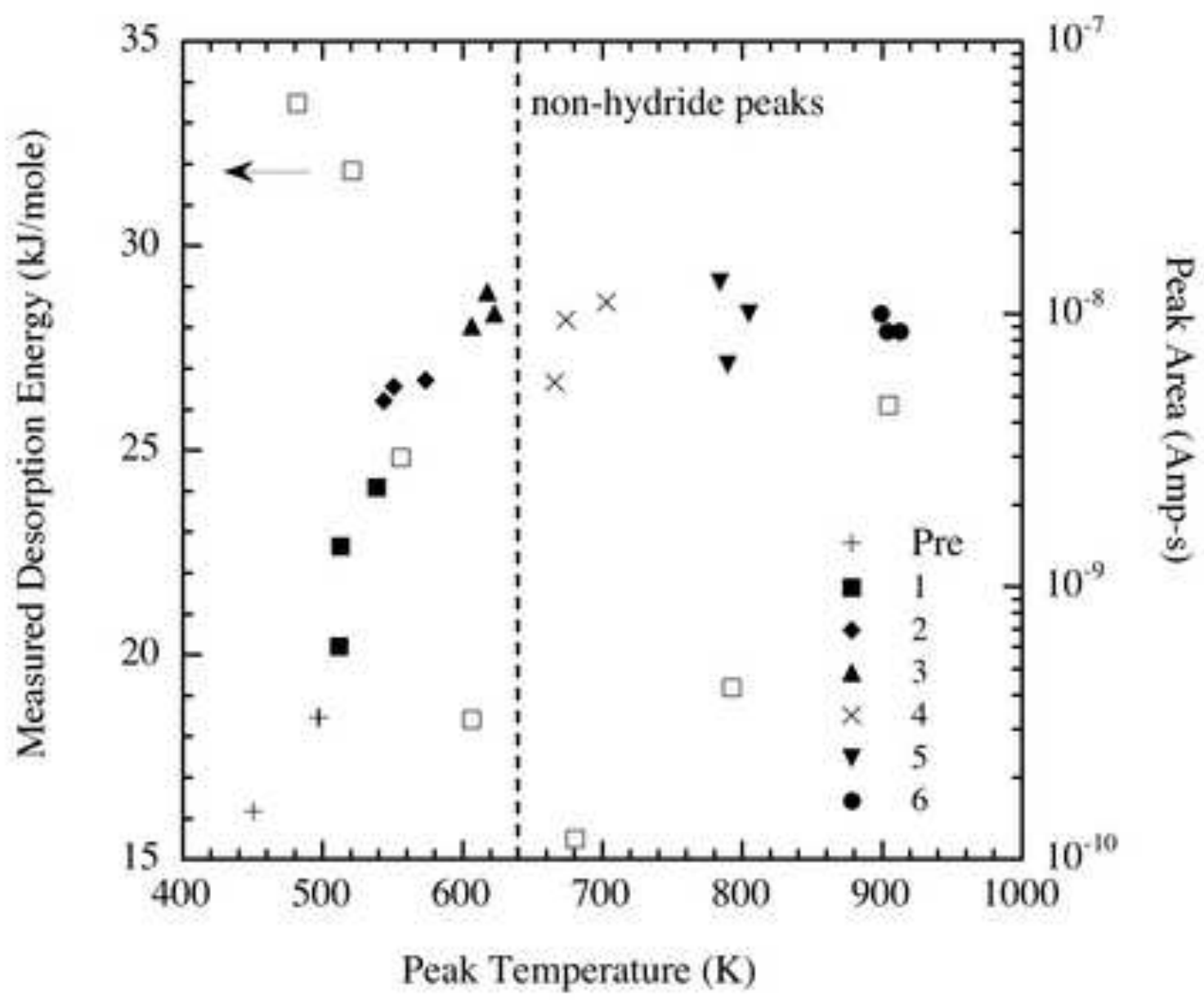

